\title{
Several kinds of oscillations in forced Liénard equations
}

Joël Blot ${ }^{*}$, Souhila Boudjema² and Philippe Cieutat ${ }^{3}$

${ }^{\text {*Correspondence: }}$

blot@univ-paris1.fr

${ }^{1}$ Laboratoire SAMM EA 4543

Université Paris 1

Panthéon-Sorbonne, centre P.M.F.

90 rue de Tolbiac, Paris cedex 13,

75634, France

Full list of author information is

available at the end of the article

\begin{abstract}
Near an equilibrium we study the existence of asymptotically a.p. (almost periodic), asymptotically a.a. (almost automorphic), pseudo a.p., pseudo a.a., weighed pseudo a.p. and weighed pseudo a.a. solutions of Liénard differential equations in the form $x^{\prime \prime}(t)+f(x(t), p) \cdot x^{\prime}(t)+g(x(t), p)=e_{p}(t)$, where the forcing term possesses a similar nature, and where $p$ is a parameter in a Banach space. We use a perturbation method around an equilibrium. We also study two special cases of the previous family of equations that are $x^{\prime \prime}(t)+f(x(t)) \cdot x^{\prime}(t)+g(x(t))=e(t)$ and $x^{\prime \prime}(t)+f(x(t), q) \cdot x^{\prime}(t)+g(x(t), q)=e(t)$.
\end{abstract}

MSC: $34 C 27 ; 34 C 99 ; 47 J 07$

Keywords: asymptotically almost periodic functions; asymptotically almost automorphic functions; pseudo almost periodic functions; pseudo almost automorphic functions; weighted pseudo almost periodic functions; weighted pseudo almost automorphic functions; Liénard equations

\section{Introduction}

We consider the following family of forced Liénard equations:

$$
x^{\prime \prime}(t)+f(x(t), p) \cdot x^{\prime}(t)+g(x(t), p)=e_{p}(t),
$$

where $P$ is a Banach space, $f: \mathbb{R} \times P \rightarrow \mathbb{R}$ and $g: \mathbb{R} \times P \rightarrow \mathbb{R}$ are two functions for each $p \in P$ and $e_{p}: \mathbb{R} \rightarrow \mathbb{R}$ is a function.

When $e_{p}$ is almost periodic in the Bohr sense (respectively almost automorphic), in [1], Theorem 3.1 (respectively Theorem 3.2), we have proven the existence of an almost periodic (respectively almost automorphic) solution $x_{p}$ near an equilibrium, by using the perturbation method in the setting of Nonlinear Functional Analysis.

In the present paper, we extend this result to the frameworks of asymptotically almost periodic, asymptotically almost automorphic, pseudo almost periodic, pseudo almost automorphic, weighted pseudo almost periodic and weighted pseudo almost automorphic functions.

We also consider two special cases of $(\mathcal{E}, p)$, which are

$$
\begin{aligned}
& x^{\prime \prime}(t)+f_{1}(x(t)) \cdot x^{\prime}(t)+g_{1}(x(t))=e(t), \\
& x^{\prime \prime}(t)+f_{2}(x(t), q) \cdot x^{\prime}(t)+g_{2}(x(t), q)=e(t) .
\end{aligned}
$$

๑ 2013 Blot et al.; licensee Springer. This is an Open Access article distributed under the terms of the Creative Commons Attribution License (http://creativecommons.org/licenses/by/2.0), which permits unrestricted use, distribution, and reproduction in any medium, provided the original work is properly cited. 
On the existence of such solutions for the two previous cases, we obtain similar results as the one obtained on $(\mathcal{E}, p)$.

Martínez-Amores and Torres in [2], then Campos and Torres in [3] described the dynamics of equation $(\mathcal{F}, e)$ in the periodic case, namely the forcing term $e$ is periodic. Then Cieutat extended these results to the almost periodic case in [4], Ait Dads, Cieutat and Lhachimi did to the pseudo almost periodic case in [5], and Cieutat, Fatajou and N'Guérékata did to the almost automorphic case in [6].

For almost periodic solutions of second-order differential systems, results on the differentiable dependence were established in [7] by Blot, Cieutat and Mawhin.

Our approach of the problem is to transform it into a nonlinear equation with parameters in Banach function spaces, and to apply the implicit function theorem of the differential calculus in Banach spaces. To realize our aim, we use the Nemytskii operators (also called superposition operators) and state some properties on these operators. Then we establish, for a linear differential equation in a Banach space, a result on the existence and uniqueness of the solutions described above. We also extend the well-know result on the almost periodicity of the derivative of an almost periodic function to the weighted pseudo almost periodic and weighted pseudo almost automorphic cases.

Now we give a brief description of the contents of the paper. In Section 2, we fix our notation and we recall some definitions. In Section 3, we establish some results on the differentiation of weighted pseudo almost periodic functions and weighted pseudo almost automorphic functions, then on a linear differential equation and on the Nemytskii operators. In Section 4, we state the main theorem (Theorem 4.1) and we give the proof of this theorem. In Section 5, we establish two corollaries (Corollary 5.1 and Corollary 5.2) of the two special cases of $(\mathcal{E}, p)$.

\section{Notation}

When $X$ and $Y$ are Banach spaces, $\mathcal{L}(X, Y)$ stands for the space of all bounded linear operators from $X$ into $Y, C^{0}(X, Y)$ (respectively $C^{1}(X, Y)$, respectively $C^{2}(X, Y)$ ) stands for the space of continuous (respectively Fréchet continuously differentiable, respectively twice Fréchet continuously differentiable) functions from $X$ into $Y$.

$B C^{0}(\mathbb{R}, X)$ stands for the space of bounded continuous functions from $\mathbb{R}$ into $X$. We also define $B C^{1}(\mathbb{R}, X):=\left\{u \in C^{1}(X, Y): u, u^{\prime} \in B C^{0}(\mathbb{R}, X)\right\}$ and $B C^{2}(\mathbb{R}, X):=\left\{u \in C^{2}(X, Y)\right.$ : $\left.u, u^{\prime}, u^{\prime \prime} \in B C^{0}(\mathbb{R}, X)\right\}$. Endowed with the norm $\|u\|_{\infty}:=\sup \{\|u(t)\|: t \in \mathbb{R}\}$ (respectively $\|u\|_{B C^{1}}:=\|u\|_{\infty}+\left\|u^{\prime}\right\|_{\infty}$, respectively $\left.\|u\|_{B C^{2}}:=\|u\|_{\infty}+\left\|u^{\prime}\right\|_{\infty}+\left\|u^{\prime \prime}\right\|_{\infty}\right), B C^{0}(\mathbb{R}, X)$ (respectively $B C^{1}(\mathbb{R}, X)$, respectively $B C^{2}(\mathbb{R}, X)$ ) is a Banach space.

A function $u \in B C^{0}(\mathbb{R}, X)$ is called an almost periodic function (in the Bohr sense) when it satisfies the following criterion (due to Bochner): $\{u(\cdot+r): r \in \mathbb{R}\}$ is relatively compact in $B C^{0}(\mathbb{R}, X) \cdot A P^{0}(X)$ denotes the space of almost periodic functions from $\mathbb{R}$ into $X$. Endowed with the norm $\|\cdot\|_{\infty}, A P^{0}(X)$ is a Banach space which is invariant by translation [8], that is to mean that $[t \mapsto u(t+\tau)] \in A P^{0}(X)$ for all $\tau \in \mathbb{R}$, when $u \in A P^{0}(X)$.

A function $u \in B C^{0}(\mathbb{R}, X)$ is called an almost automorphic function (in the Bochner sense) when, for all real sequence $\left(s_{n}\right)_{n}$, there exists a subsequence $\left(t_{n}\right)_{n}$ of $\left(s_{n}\right)_{n}$ such that for all $t \in \mathbb{R}, \lim _{n \rightarrow \infty} u\left(t+t_{n}\right)=v(t)$ exists in $X$, and for all $t \in \mathbb{R}, \lim _{n \rightarrow \infty} v\left(t-t_{n}\right)=u(t)$ exists. $A A^{0}(X)$ denotes the space of almost automorphic functions from $\mathbb{R}$ into $X$. Endowed with the norm $\|\cdot\|_{\infty}, A A^{0}(X)$ is a Banach space which is invariant by translation [9].

We also consider the following other function spaces which one can find in [10]: 
- $C_{0}(X):=\left\{u \in B C(\mathbb{R}, X): \lim _{|t| \rightarrow \infty}\|u(t)\|=0\right\}$.

- $A A P^{0}(X):=A P^{0}(X) \oplus C_{0}(X)$ the space of asymptotically almost periodic functions [8].

- $A A A^{0}(X):=A A^{0}(X) \oplus C_{0}(X)$ the space of asymptotically almost automorphic functions [9].

- $P_{0}(X):=\left\{u \in B C(\mathbb{R}, X): \lim _{T \rightarrow \infty} \frac{1}{2 T} \int_{-T}^{+T}\|u(s)\| d s=0\right\}$.

- $P A P^{0}(X):=A P^{0}(X) \oplus P_{0}(X)$ the space of pseudo almost periodic functions [11, 12].

- $P A A^{0}(X):=A A^{0}(X) \oplus P_{0}(X)$ the space of pseudo almost automorphic functions $[13,14]$.

Endowed with the norm $\|\cdot\|_{\infty}, A A P^{0}(X), A A A^{0}(X), P A P^{0}(X)$ and $P A A^{0}(X)$ are Banach spaces which are invariant by translation (cf. respectively $[8,9,11]$ and [14]).

Let $\mathcal{L}_{\text {loc }}^{1}(\mathbb{R},(0, \infty))$ be the set of all functions $\rho: \mathbb{R} \rightarrow(0, \infty)$ which are positive and locally Lebesgue-integrable over $\mathbb{R}$. For a given $r \in(0, \infty)$ and for each $\rho \in \mathcal{L}_{\text {loc }}^{1}(\mathbb{R},(0, \infty))$, we set $m(r, \rho):=\int_{-r}^{+r} \rho(x) d x$.

We define the following spaces:

- $\mathcal{U}_{\infty}:=\left\{\rho \in \mathcal{L}_{\text {loc }}^{1}(\mathbb{R},(0, \infty)): \lim _{r \rightarrow \infty} m(r, \rho)=\infty\right\}$

- $\mathcal{U}_{T}:=\left\{\rho \in \mathcal{U}_{\infty}: \rho\right.$ satisfies $\left.(\mathrm{H})\right\}$, where $(\mathrm{H})$ is the following condition due to [15]:

(H) For all $\tau \in \mathbb{R}$, there exist $\beta>0$ and a bounded interval $I$ such that $\rho(t+\tau) \leq \beta \cdot \rho(t)$ a.e. $t \in \mathbb{R} \backslash I$,

which is equivalent to $\forall \tau \in \mathbb{R}, \lim \sup _{|t| \rightarrow \infty} \frac{\rho(t+\tau)}{\rho(t)}<\infty(c f$. Remark 3.4 in [15] or Remark 3.1 in [16]).

For $\rho \in \mathcal{U}_{\infty}$, we consider the following spaces:

- $P_{0}(X, \rho):=\left\{u \in B C(\mathbb{R}, X): \lim _{r \rightarrow \infty} \frac{1}{m(r, \rho)} \int_{-r}^{+r}\|u(s)\| \rho(s) d s=0\right\}$.

- $W P A P^{0}(X, \rho):=A P^{0}(X) \oplus P_{0}(X, \rho)$ the space of weighted pseudo almost periodic functions $[17,18]$.

- $W P A A^{0}(X, \rho):=A A^{0}(X) \oplus P_{0}(X, \rho)$ the space of weighted pseudo almost automorphic functions [19].

Let $\mu$ be a positive measure on $\mathfrak{B}$ ( $\mathfrak{B}$ is a Lebesgue $\sigma$-field on $\mathbb{R}$ ) satisfying $\mu(\mathbb{R})=\infty$ and $\mu([a, b])<\infty$ for all $a \leq b$. A function $u$ is called $\mu$-pseudo almost periodic (respectively $\mu$-pseudo almost automorphic) if $u=g+\phi$, where $g \in A P^{0}(X)$ (respectively $g \in A A^{0}(X)$ ) and $\phi$ is a function satisfying

$$
\lim _{r \rightarrow \infty} \frac{1}{\mu([-r, r])} \int_{[-r, r]}\|\phi(s)\| d \mu(s) d s=0,
$$

where $\mu([-r, r])$ is the measure of the set $[-r, r]$. The set of such functions is denoted by $P A P(\mathbb{R}, X, \mu)$ (respectively $P A A(\mathbb{R}, X, \mu)) c f$. [15] (respectively [16]). When the measure $\mu$ satisfies the following condition:

(C) For all $\tau \in \mathbb{R}$, there exist $\beta>0$ and a bounded interval $I$ such that $\mu(\{a+\tau: a \in B\}) \leq \beta \cdot \mu(B)$ for all $B \in \mathfrak{B}$ satisfying $B \cap I=\emptyset$,

then the set $P A P(\mathbb{R}, X, \mu)$ (respectively $P A A(\mathbb{R}, X, \mu)$ ) is a Banach space which is invariant by translation, $c f$. Corollary 2.31 and Theorem 3.3 in [15] (respectively Theorem 4.9 and Theorem 3.5 in [16]).

The space $\operatorname{WPAP}^{0}(X, \rho)$ (respectively $\left.\operatorname{WPAA}^{0}(X, \rho)\right)$ is a special case of the space $P A P(\mathbb{R}, X, \mu)$ (respectively $P A A(\mathbb{R}, X, \mu))$ in the following sense: $W_{P A P^{0}}(X, \rho)=P A P(\mathbb{R}, X$, $\mu)\left(\right.$ respectively $W P A A^{0}(X, \rho)=P A A(\mathbb{R}, X, \mu)$ ), where the measure $\mu$ is absolutely continuous with respect to the Lebesgue measure and its Radon-Nikodym derivative is $\rho=\frac{d \mu}{d t}$. The function $\rho$ satisfies hypothesis $(\mathrm{H})$ if and only if the measure $\mu$ satisfies condition $(\mathrm{C})$, 
cf. Remark 3.4 in [15] (respectively Remark 3.1 in [16]). Consequently, when $\rho \in \mathcal{U}_{T}$, the spaces $\operatorname{WPAP}^{0}(X, \rho)$ and $W P A A^{0}(X, \rho)$ are Banach spaces which are invariant by translation.

$E^{0}(X)$ denotes one the following spaces: $A A P^{0}(X), A A A^{0}(X), P A P^{0}(X), P A A^{0}(X)$, $\operatorname{WPAP}^{0}(X, \rho)$, or $W P A A^{0}(X, \rho)$ endowed with the norm $\|\cdot\|_{\infty}$. We have $E^{0}(X) \subset$ $B C^{0}(\mathbb{R}, X)$, and when $\rho \in \mathcal{U}_{T}, E^{0}(X)$ is a Banach space which is invariant by translation. $E^{1}(X)$ denotes the space of functions $u \in B C^{1}(\mathbb{R}, X)$ such that $u, u^{\prime} \in E^{0}(X)$. Endowed with the norm $\|\cdot\|_{B C^{1}}, E^{1}(X)$ is a Banach space. $E^{2}(X)$ denotes the space of the functions $u \in B C^{2}(\mathbb{R}, X)$ such that $u, u^{\prime}, u^{\prime \prime} \in E^{0}(X)$. Endowed with the norm $\|\cdot\|_{B C^{2}}, E^{2}(X)$ is a Banach space.

\section{Preliminary results}

For the proof of the main result, we need the following lemmas.

Lemma 3.1 Let $\rho \in \mathcal{U}_{T}$ and $u \in C^{1}(\mathbb{R}, X) \cap E^{0}(X)$. If the derivative $u^{\prime}$ is uniformly continuous, then $u^{\prime} \in E^{0}(X)$, thus $u \in E^{1}(X)$.

Remark 3.2 Lemma 3.1 is well know for $A P^{0}(X), A A^{0}(X)$. In the scalar case, for $P A P^{0}(X)$, this result is proved in [12], Corollary 5.6, p.59.

Proof Consider the function $g_{n}: \mathbb{R} \rightarrow X$ defined by $g_{n}(t):=n\left(f\left(t+\frac{1}{n}\right)-f(t)\right)$ for $n \in \mathbb{N} \backslash$ $\{0\}$. Since $E^{0}(X)$ is a translation invariant vectorial space, then $g_{n} \in E^{0}(X)$. The equality $g_{n}(t)-f^{\prime}(t)=n \int_{0}^{\frac{1}{n}}\left(f^{\prime}(t+s)-f^{\prime}(t)\right) d s$ shows that the uniform continuity of $f^{\prime}$ implies that the sequence $\left(g_{n}\right)_{n}$ with values in the Banach space $E^{0}(X)$ converges uniformly to $f^{\prime}$ on $\mathbb{R}$. Then $f^{\prime} \in E^{0}(X)$, and from the definition of $E^{1}(X)$, we obtain $f \in E^{1}(X)$.

Lemma 3.3 Let $\rho \in \mathcal{U}_{T}$ and $A \in \mathcal{L}(X, X)$. If the spectrum $\sigma(A)$ of $A$ does not intersect the imaginary axis, then for all $h \in E^{0}(X)$, there exists a unique solution in $E^{0}(X)$ of the differential equation

$$
u^{\prime}(t)=A u(t)+h(t)
$$

Moreover, the solution $u$ is in $E^{1}(X)$.

Proof Applying Theorem 4.1, p.81 in [20] (or Theorem 4 in [21]), Equation (3.1) admits a unique bounded solution on $\mathbb{R}$ which is given by the formula

$$
u(t)=\int_{-\infty}^{\infty} G(t-s) h(s) d s
$$

where $G$ is the principal Green function for Equation (3.1). The Green function $G: \mathbb{R} \rightarrow$ $\mathcal{L}(X, X)$ is continuous on $\mathbb{R}-\{0\}$, and there exist $M \geq 1$ and $\omega>0$ such that $\|G(t)\| \leq$ $M \exp (-\omega|t|)$ for all $t \in \mathbb{R}$.

Now we prove that the bounded solution $u$ defined by (3.2) belongs to $E^{0}(X)$.

When $E^{0}(X)=A P^{0}(X)$ (respectively $E^{0}(X)=A A^{0}(X)$ ), this result is a straightforward consequence of Theorem 3.8 in [15] (respectively Theorem 3.9 in [16]) and when $E^{0}(X)=$ $C_{0}(X)$, this result is proved in [21], Proposition 3. Then we deduce the result for $E^{0}(X)=$ $A A P^{0}(X)\left(\right.$ respectively $\left.E^{0}(X)=A A A^{0}(X)\right)$. 
Note that the case of the pseudo almost periodic (respectively pseudo almost automorphic) functions is a special case of the weighted pseudo almost periodic (respectively weighted pseudo almost automorphic) functions by taking $\rho(t):=1$ for all $t \in \mathbb{R}$; remark that the associated measure is exactly the Lebesgue measure.

And so it suffices to prove the cases of weighted pseudo almost periodic functions and of weighted pseudo almost automorphic functions.

When $E^{0}(X)=\operatorname{WPAP}^{0}(X, \rho)$ (respectively $\left.E^{0}(X)=W P A A^{0}(X, \rho)\right)$, this result is a straightforward consequence of Theorem 3.8 in [15] (respectively Theorem 3.9 in [16]). Consequently, $u \in E^{0}(X)$, and from the definition of $E^{0}(X)$, we deduce that $[t \mapsto A u(t)] \in$ $E^{0}(X)$. Since $u$ satisfies Equation (3.1), then $u^{\prime} \in E^{0}(X)$, and from the definition of $E^{1}(X)$, we obtain $u \in E^{1}(X)$.

Lemma 3.4 Let $X$ and $Y$ be two finite-dimensional Banach spaces, and let $\phi: X \rightarrow Y$ be a continuous mapping. Then the Nemytskii operator $N_{\phi}: E^{0}(X) \rightarrow E^{0}(Y)$, defined by $N_{\phi}(u):=[t \mapsto \phi(u(t))]$, is continuous.

Remark 3.5 Contrary to the asymptotically almost periodic case and, in particular, for the almost periodic case, when the dimension of the Banach spaces $X$ and $Y$ is infinite, Lemma 3.4 does not hold for the pseudo almost periodic case, and thus for the weighted pseudo almost periodic case, without additional assumptions. This is due to the fact that the range of a pseudo almost periodic function is only bounded, but not relatively compact, contrary to the asymptotically almost periodic case. This last observation still holds when the word almost periodic is replaced by almost automorphic.

Proof When $E^{0}(X)=A A P^{0}(X)$ and $E^{0}(Y)=A A P^{0}(Y)$, replacing $\mathbb{R}_{+}$by $\mathbb{R}$, this result is a variation of Theorem 8.4 in [22].

When $E^{0}(X)=A A A^{0}(X)$ and $E^{0}(Y)=A A A^{0}(Y)$, replacing $\mathbb{R}_{+}$by $\mathbb{R}$, the inclusion $N_{\phi}\left(A A A^{0}(X)\right) \subset A A A^{0}(Y)$ is a variation of Theorem 2.15 in [9]. Moreover, using Lemma 1 in [23], we know that $N_{\phi}: B C^{0}(\mathbb{R}, X) \rightarrow B C^{0}(\mathbb{R}, Y)$ is continuous, and so its restriction to $A A A^{0}(X)$ is also continuous.

When $E^{0}(X)=P A P^{0}(X)$ and $E^{0}(Y)=P A P^{0}(Y)$ (respectively $E^{0}(X)=P A A^{0}(X)$ and $E^{0}(Y)=P A A^{0}(Y)$ ), this result is a straightforward consequence of Theorem 4.1 (respectively Theorem 4.2) in [24].

When $E^{0}(X)=W P A P^{0}(X, \rho)$ and $E^{0}(Y)=\operatorname{WPAP}^{0}(Y, \rho)\left(\right.$ respectively $E^{0}(X)=W P A A^{0}(X$, $\rho$ ) and $E^{0}(Y)=\operatorname{WPA}^{0}(Y, \rho)$ ), using Corollary 4.12 in [15] (respectively Corollary 5.10 in [16]), we know that $N_{\phi}\left(W P A P^{0}(X, \rho)\right) \subset W P A P^{0}(Y, \rho)\left(\right.$ respectively $N_{\phi}\left(W P A A^{0}(X, \rho)\right) \subset$ $\left.W P A P^{0}(Y, \rho)\right)$. Moreover, using Lemma 1 in [23], we know that $N_{\phi}: B C^{0}(\mathbb{R}, X) \rightarrow$ $B C^{0}(\mathbb{R}, Y)$ is continuous, and so its restriction to $\operatorname{WPAP}^{0}(X, \rho)$ (respectively $\operatorname{WPA} A^{0}(X, \rho)$ ) is also continuous.

Lemma 3.6 Let $X$ and $Y$ be two finite-dimensional Banach spaces, and let $\phi: X \rightarrow$ $Y$ be a continuously Fréchet-differentiable mapping. Then the Nemytskii operator $N_{\phi}$ : $E^{0}(X) \rightarrow E^{0}(Y)$ is continuously Fréchet-differentiable on $E^{0}(X)$, and we have $D N_{\phi}(u) v=$ $[t \mapsto D \phi(u(t)) v(t)]$ for all $u, v \in E^{0}(X)$.

Proof When $E^{0}(X)=A A P^{0}(X)$ and $E^{0}(Y)=A A P^{0}(Y)$, replacing $\mathbb{R}_{+}$by $\mathbb{R}$, this result is a variation of Theorem 8.5 in [22]. 
When $E^{0}(X)=A A A^{0}(X)$ and $E^{0}(Y)=A A A^{0}(Y)$, using Lemma 1 in [23], we know that $N_{\phi}: B C^{0}(\mathbb{R}, X) \rightarrow B C^{0}(\mathbb{R}, Y)$ is of class $C^{1}$ and that we have $D N_{\phi}(u) h=[t \mapsto$ $D \phi(u(t)) \cdot h(t)]$ when $u, h \in B C^{0}(\mathbb{R}, X)$. Now, using Theorem 2.15 in [9], we know that $N_{\phi}\left(A A A^{0}(X)\right) \subset A A A^{0}(Y)$ and that $D \phi \circ u \in A A A^{0}(\mathcal{L}(X, Y))$ when $u \in A A A^{0}(X)$. And so $N_{\phi} \in C^{1}\left(A A A^{0}(X), A A A^{0}(Y)\right)$ and the announced formula for its Fréchet-differential is proven.

As in the proof of Lemma 3.3, the case of $P A P^{0}(X)$ (respectively $\left.P A A^{0}(X)\right)$ is a corollary of the case $\operatorname{WPAP}^{0}(X, \rho)$ (respectively $\operatorname{WPAA}^{0}(X, \rho)$ ). And so it suffices to prove the cases of the weighted pseudo almost periodic functions and of the weighted pseudo almost automorphic functions.

To prove the result in the case where $E^{0}(X)=\operatorname{WPAP}^{0}(X, \rho)$ and $E^{0}(Y)=\operatorname{WPAP}^{0}(Y, \rho)$ (respectively $E^{0}(X)=W P A A^{0}(X, \rho)$ and $E^{0}(Y)=W P A A^{0}(Y, \rho)$ ), note that, using Lemma 1 in [23], we know that $N_{\phi}: B C^{0}(\mathbb{R}, X) \rightarrow B C^{0}(\mathbb{R}, Y)$ is of class $C^{1}$ and that we have $D N_{\phi}(u) h=[t \mapsto D \phi(u(t)) \cdot h(t)]$ when $u, h \in B C^{0}(\mathbb{R}, X)$. Now, using Corollary 4.12 in [15] (respectively Corollary 5.10 in [16]), we know that $N_{\phi}\left(\operatorname{WPAP}^{0}(X, \rho)\right) \subset \operatorname{WPAP}^{0}(Y, \rho)$ (respectively $\left.N_{\phi}\left(W P A A^{0}(X, \rho)\right) \subset W P A A^{0}(Y, \rho)\right)$ and that $D \phi \circ u \in W P A P^{0}(\mathcal{L}(X, Y), \rho)$ (respectively $D \phi \circ u \in W P A A^{0}(\mathcal{L}(X, Y), \rho)$ ) when $u \in \operatorname{WPAP}^{0}(X, \rho)$ (respectively $\left.\operatorname{WPAA}^{0}(X, \rho)\right)$.

Consequently, we obtain $N_{\phi} \in C^{1}\left(\operatorname{WPAP}^{0}(X, \rho), \operatorname{WPAP}^{0}(Y, \rho)\right)$ (respectively $N_{\phi} \in$ $\left.C^{1}\left(W_{P A A^{0}}(X, \rho), W P A A^{0}(Y, \rho)\right)\right)$ and the announced formula for its Fréchet-differential is proven.

Lemma 3.7 Let $\rho \in \mathcal{U}_{T}, p \in P$ and $e_{p} \in E^{0}(\mathbb{R})$. If $x$ is a solution of $(\mathcal{E}, p)$ in $E^{0}(\mathbb{R})$, that is, $x \in C^{2}(\mathbb{R}, \mathbb{R}) \cap E^{0}(\mathbb{R})$ and $x$ satisfies $(\mathcal{E}, p)$, then $x \in E^{2}(\mathbb{R})$.

Proof Lemma 3.2 in [4] asserts that if $x$ is a solution of $(\mathcal{E}, p)$ in $B C^{0}(\mathbb{R}, \mathbb{R})$, then $x \in$ $B C^{2}(\mathbb{R}, \mathbb{R})$, therefore the derivative $x^{\prime}$ is uniformly continuous, and by help of Lemma 3.1, we obtain $x \in E^{1}(\mathbb{R})$. By using Lemma 3.4, the functions $[t \mapsto f(x(t), p)]$ and $[t \mapsto g(x(t), p)]$ are in $E^{0}(\mathbb{R})$. Applying again Lemma 3.4 to $\left[t \mapsto\left(f(x(t), p), x^{\prime}(t)\right)\right] \in E^{0}\left(\mathbb{R}^{2}\right)$ and using the continuous function $\phi: \mathbb{R}^{2} \rightarrow \mathbb{R}$ defined by $\phi(r, s):=r \cdot s$, we obtain that $[t \mapsto f(x(t), p)$. $\left.x^{\prime}(t)\right] \in E^{0}(\mathbb{R})$. Since $x^{\prime \prime}(t)=e_{p}(t)-f(x(t), p) \cdot x^{\prime}(t)-g(x(t), p)$, then $x^{\prime \prime} \in E^{0}(\mathbb{R})$, and from the definition of $E^{2}(\mathbb{R})$, we obtain that $x \in E^{2}(\mathbb{R})$.

\section{The main result}

First we announce the main result of the paper.

Theorem 4.1 Let $\rho \in \mathcal{U}_{T}$. Under the following assumptions:

(A1) $f, g \in C^{1}(\mathbb{R} \times P, \mathbb{R})$,

(A2) $g(0,0)=0$,

(A3) $p \mapsto e_{p} \in C^{1}\left(P, E^{0}(\mathbb{R})\right)$ and $e_{0}=0$,

(A4) $f(0,0) \neq 0$ when $f(0,0)^{2}<4 \frac{\partial g(0,0)}{\partial x}$, and $\frac{\partial g(0,0)}{\partial x} \neq 0$ when $f(0,0)^{2} \geq 4 \frac{\partial g(0,0)}{\partial x}$,

there exist a neighborhood $\mathcal{U}$ of 0 in $E^{2}(\mathbb{R})$, a neighborhood $\mathcal{V}$ of 0 in $P$ and a $C^{1}$-mapping $p \mapsto \underline{x}[p]$ from $\mathcal{V}$ into $\mathcal{U}$ which satisfies the following conditions:

(i) $\underline{x}[0]=0$,

(ii) for all $p \in \mathcal{V}, \underline{x}[p]$ is a solution of $(\mathcal{E}, p)$ in $E^{0}(\mathbb{R})$,

(iii) if $x \in \mathcal{U}$ is a solution of $(\mathcal{E}, p)$ in $E^{0}(\mathbb{R})$ with $p \in \mathcal{V}$, then $x=\underline{x}[p]$. 
To prove Theorem 4.1, we define the operator $\Phi: E^{2}(\mathbb{R}) \times P \rightarrow E^{0}(\mathbb{R})$ by setting

$$
\Phi(x, p):=\left[t \mapsto x^{\prime \prime}(t)+f(x(t), p) \cdot x^{\prime}(t)+g(x(t), p)-e_{p}(t)\right]
$$

when $x \in E^{2}(\mathbb{R})$ and $p \in P$.

Let $p \in P$. By using Lemma 3.7, we deduce that $x \in E^{2}(\mathbb{R})$ satisfies $\Phi(x, p)=0$ if and only if $x$ is a solution of $(\mathcal{E}, p)$ in $E^{0}(\mathbb{R})$.

Under (A2) and (A3), note that 0 is a solution of $(\mathcal{E}, 0)$ in $E^{2}(\mathbb{R})$, and so the following equality holds:

$$
\Phi(0,0)=0
$$

Lemma 4.2 Under (A1)-(A3), the operator $\Phi$ is well defined and it is of class $C^{1}$ on $E^{2}(\mathbb{R}) \times P$. Moreover, the partial differential of $\Phi$ with respect to the first variable, at the point $(x, p)=(0,0)$, is given by

$$
D_{x} \Phi(0,0) y=\left[t \mapsto y^{\prime \prime}(t)+f(0,0) \cdot y^{\prime}(t)+\frac{\partial g(0,0)}{\partial x} \cdot y(t)\right]
$$

when $y \in E^{2}(\mathbb{R})$.

Proof First we introduce linear operators: $\frac{d^{2}}{d t^{2}}: E^{2}(\mathbb{R}) \rightarrow E^{0}(\mathbb{R})$ defined by $\frac{d^{2}}{d t^{2}} x:=x^{\prime \prime}, \frac{d}{d t}$ : $E^{1}(\mathbb{R}) \rightarrow E^{0}(\mathbb{R})$ defined by $\frac{d}{d t} x:=x^{\prime}, \operatorname{in}_{1}: E^{2}(\mathbb{R}) \rightarrow E^{1}(\mathbb{R})$ defined by $\operatorname{in}_{1}(x):=x$ and $\operatorname{in}_{2}:$ $E^{2}(\mathbb{R}) \rightarrow E^{0}(\mathbb{R})$ defined by $\operatorname{in}_{2}(x):=x$. Since $\left\|\frac{d^{2}}{d t^{2}} x\right\|_{\infty} \leq\|x\|_{B C^{2}},\left\|\operatorname{in}_{1}(x)\right\|_{B C^{1}} \leq\|x\|_{B C^{2}}$ and $\left\|\operatorname{in}_{2}(x)\right\|_{\infty} \leq\|x\|_{B C^{2}}$ for all $x \in E^{2}(\mathbb{R})$, and $\left\|\frac{d}{d t} x\right\|_{\infty} \leq\|x\|_{B C^{1}}$ for all $x \in E^{1}(\mathbb{R})$, these linear operators are continuous; and consequently, the following assertion holds:

$$
\frac{d^{2}}{d t^{2}}, \frac{d}{d t}, \mathrm{in}_{1}, \mathrm{in}_{2} \text { are of class } C^{1}
$$

Now we define the Nemytskii operators build on the functions $f$ and $g: N_{f}: E^{0}(\mathbb{R}) \times$ $E^{0}(P) \rightarrow E^{0}(\mathbb{R})$ defined by $N_{f}(x, p):=[t \mapsto f(x(t), p(t))]$ and $N_{g}: E^{0}(\mathbb{R}) \times E^{0}(P) \rightarrow E^{0}(\mathbb{R})$ defined by $N_{g}(x, p):=[t \mapsto g(x(t), p(t))]$. By using Lemma 3.6, $N_{f}$ and $N_{g}$ are of class $C^{1}$ on $E^{0}(\mathbb{R}) \times E^{0}(P)$ assimilated to $E^{0}(\mathbb{R} \times P)$, and Lemma 3.6 provides formulas for the differentials of these Nemytskii operators:

$$
\begin{aligned}
& D_{x} N_{f}(x, p) y=\left[t \mapsto \frac{\partial f(x(t), p(t))}{\partial x} \cdot y(t)\right] \\
& D_{x} N_{g}(x, p) y=\left[t \mapsto \frac{\partial g(x(t), p(t))}{\partial x} \cdot y(t)\right]
\end{aligned}
$$

for all $x, y \in E^{0}(\mathbb{R})$.

We can assimilate a point $p \in P$ to the constant function $t \mapsto p$ that belongs to $E^{0}(P)$, which permits us to look at $P$ as a closed vector subspace of $E^{0}(P)$. Then we can consider the following restrictions of the operators $N_{f}$ and $N_{g}: S_{f}: E^{0}(\mathbb{R}) \times P \rightarrow E^{0}(\mathbb{R})$ defined by $S_{f}(x, p):=[t \mapsto f(x(t), p)]$ and $S_{g}: E^{0}(\mathbb{R}) \times P \rightarrow E^{0}(\mathbb{R})$ defined by $S_{g}(x, p):=[t \mapsto g(x(t), p)]$ when $x \in E^{0}(\mathbb{R})$ and $p \in P$. 
Since the restriction of a $C^{1}$-mapping to a Banach subspace is also a $C^{1}$-mapping, a straightforward consequence of the continuous differentiability of $N_{f}$ and of $N_{g}$ is that $S_{f}$ and $S_{g}$ are of class $C^{1}$, and the consequences of (4.3) and (4.4) are the following formulas:

$$
D_{x} S_{f}(x, p) y=\left[t \mapsto \frac{\partial f(x(t), p)}{\partial x} \cdot y(t)\right], \quad D_{x} S_{g}(x, p) y=\left[t \mapsto \frac{\partial g(x(t), p)}{\partial x} \cdot y(t)\right]
$$

for all $x, y \in E^{0}(\mathbb{R})$ for all $p \in P$.

Now we consider the following operators: $\pi_{1}: E^{2}(\mathbb{R}) \times P \rightarrow E^{2}(\mathbb{R})$ defined by $\pi_{1}(x, p):=$ $x, \pi_{2}: E^{2}(\mathbb{R}) \times P \rightarrow P$ defined by $\pi_{2}(x, p):=p$ and $B: \mathbb{R} \times \mathbb{R} \rightarrow \mathbb{R}$ defined by $B(r, s):=r \cdot s$. We consider the Nemystkii operator build on $B, N_{B}: E^{0}(\mathbb{R}) \times E^{0}(\mathbb{R}) \rightarrow E^{0}(\mathbb{R})$ defined by $N_{B}(u, v):=[t \mapsto u(t) \cdot v(t)=B(u(t), v(t))]$ and $C: E^{2}(\mathbb{R}) \times P \rightarrow E^{0}(\mathbb{R})$ defined by $C(x, p):=$ $-e_{p}$.

Since $\pi_{1}$ and $\pi_{2}$ are linear continuous, they are of class $C^{1}$. Since $B$ is bilinear continuous, it is of class $C^{1}$; and consequently, using Lemma 3.6, $N_{B}$ is of class $C^{1}$ on $E^{0}(\mathbb{R}) \times E^{0}(\mathbb{R})$. Denoting by $\varepsilon: P \rightarrow E^{0}(\mathbb{R})$ the mapping $p \mapsto e_{p}, \varepsilon$ is of class $C^{1}$ after (A3), and $C=-\varepsilon \circ \pi_{2}$ is of class $C^{1}$ as a composition of $C^{1}$-mappings. And so we can assert that $\pi_{1}, \pi_{2}, N_{B}$ and $C$ are of class $C^{1}$.

Now we note that the following equality holds:

$$
\begin{aligned}
\Phi= & \frac{d^{2}}{d t^{2}} \circ \pi_{1}+N_{B} \circ\left(S_{f} \circ\left(\mathrm{in}_{2} \circ \pi_{1}, \pi_{2}\right), \frac{d}{d t} \circ\left(\mathrm{in}_{1} \circ \pi_{1}\right)\right) \\
& +S_{g} \circ\left(\mathrm{in}_{2} \circ \pi_{1}, \pi_{2}\right)+C .
\end{aligned}
$$

Since all the mappings which are present in the previous formula are of class $C^{1}$, using the usual rules of the differential calculus in Banach spaces, we obtain that $\Phi$ is of class $C^{1}$.

For all $y \in E^{2}(\mathbb{R})$, by using the classical formulas of the differential calculus in Banach spaces and (4.5), we obtain

$$
D_{x} \Phi(0,0) y=\frac{d^{2}}{d t^{2}} y+N_{B}\left(\frac{\partial f}{\partial x}(0,0) y, 0\right)+N_{B}\left(S_{f}(0,0), \frac{d}{d t}\left(\operatorname{in}_{1}(y)\right)\right)+D_{x} S_{g}(0,0) y
$$

which implies, for all $t \in \mathbb{R}$,

$$
\left(D_{x} \Phi(0,0) y\right)(t)=y^{\prime \prime}(t)+f(0,0) \cdot y^{\prime}(t)+\frac{\partial g(0,0)}{\partial x} \cdot y(t)
$$

which is the announced formula.

Lemma 4.3 Under (A1)-(A4), $D_{x} \Phi(0,0)$ is a bijection from $E^{2}(\mathbb{R})$ onto $E^{0}(\mathbb{R})$.

Proof Let $b \in E^{0}(\mathbb{R})$. We want to prove that there exists a unique $y \in E^{2}(\mathbb{R})$ such that $D_{x} \Phi(0,0) y=b$. Using the formula provided by Lemma 4.2, this equation is equivalent to saying that $y$ is a solution in $E^{2}(\mathbb{R})$ of the following second-order linear differential equation (which is a Duffing equation):

$$
y^{\prime \prime}(t)+f(0,0) \cdot y^{\prime}(t)+\frac{\partial g(0,0)}{\partial x} \cdot y(t)=b(t) .
$$


Rewriting this second-order equation in the form of a first-order system, we obtain the following equivalent differential system:

$$
X^{\prime}(t)=M X(t)+B(t)
$$

where $X(t):=\left[\begin{array}{c}y(t) \\ y^{\prime}(t)\end{array}\right], B(t):=\left[\begin{array}{c}0 \\ b(t)\end{array}\right]$, and $M:=\left[\begin{array}{cc}0 & 1 \\ -\frac{\partial g(0,0)}{\partial x} & -f(0,0)\end{array}\right]$.

For $\rho \in \mathcal{U}_{T}$ and with condition (A4), the assumptions of Lemma 3.3 are fulfilled, and we can assert that there exists a unique $X \in E^{1}\left(\mathbb{R}^{2}\right)$, which is a solution of (4.7). Therefore the first coordinate of $X$, denoted by $y$, is the unique solution of (4.6) in $E^{2}(\mathbb{R})$ since $y$ and $y^{\prime} \in E^{1}(\mathbb{R})$, and then $y$ is the unique element of $E^{2}(\mathbb{R})$ which satisfies $D_{x} \Phi(0,0) y=b$.

Proof of Theorem 4.1 By using (4.2), Lemma 4.2 and Lemma 4.3, we can use the implicit function theorem ([25], p.61) that permits us to say that there exist a neighborhood $\mathcal{U}$ of 0 in $E^{2}(\mathbb{R})$, a neighborhood $\mathcal{V}$ of 0 in $P$ and a $C^{1}$-mapping $p \mapsto \underline{x}[p]$ from $\mathcal{V}$ into $\mathcal{U}$, which satisfies the following conditions:

(a) $\underline{x}[0]=0$, that is, the condition (i) of Theorem 4.1.

(b) $\Phi(\underline{x}[p], p)=0$ for all $p \in \mathcal{V}$, that ensures that $\underline{x}[p]$ is a solution of $(\mathcal{E}, p)$ in $E^{0}(\mathbb{R})$ for all $p \in \mathcal{V}$, that is, the conclusion (ii) of Theorem 4.1.

(c) $\{(x, p) \in \mathcal{U} \times \mathcal{V}: \Phi(x, p)=0\}=\{(\underline{x}[p], p): p \in \mathcal{V}\}$ that implies the conclusion (iii) of Theorem 4.1.

And so Theorem 4.1 is proven.

\section{Special cases}

We consider the equation

$$
x^{\prime \prime}(t)+f_{1}(x(t)) \cdot x^{\prime}(t)+g_{1}(x(t))=e(t)
$$

which is a special case of $(\mathcal{E}, p)$ by taking $f(x, p)=f_{1}(x), g(x, p)=g_{1}(x)$ and $p \mapsto e_{p}$ defined as the identity operator on $P=E^{0}(\mathbb{R})$.

On the existence solution of $(\mathcal{F}, e)$ in $\mathbb{E}^{0}(\mathbb{R})$, we establish the following result.

Corollary 5.1 Let $\rho \in \mathcal{U}_{T}$. Under the following assumptions:

(A5) $f_{1}, g_{1} \in C^{1}(\mathbb{R}, \mathbb{R})$,

(A6) $g_{1}(0)=0$,

(A7) $f_{1}(0) \neq 0$ when $f_{1}(0)^{2}<4 g_{1}^{\prime}(0)$, and $g_{1}^{\prime}(0) \neq 0$ when $f_{1}(0)^{2} \geq 4 g_{1}^{\prime}(0)$,

there exist a neighborhood $\mathcal{W}$ of 0 in $E^{0}(\mathbb{R})$, a neighborhood $\mathcal{U}$ of 0 in $E^{2}(\mathbb{R})$ and a $C^{1}$-mapping $e \mapsto \underline{x}[e]$ from $\mathcal{W}$ into $\mathcal{U}$, which satisfies the following conditions:

(i) $\underline{x}[0]=0$,

(ii) for all $e \in \mathcal{W}, \underline{x}[e]$ is a solution of $(\mathcal{F}, e)$ in $\mathbb{E}^{0}(\mathbb{R})$,

(iii) if $x \in \mathcal{U}$ is a solution of $(\mathcal{F}, e)$ with $e \in \mathcal{W}$ in $E^{0}(\mathbb{R})$, then we have $x=\underline{x}[e]$.

The second special case of the equation $(\mathcal{E}, p)$ is

$$
x^{\prime \prime}(t)+f_{2}(x(t), q) \cdot x^{\prime}(t)+g_{2}(x(t), q)=e(t)
$$

when $q$ belongs to a Banach space $Q$, and by taking $p=(e, q) \in P=E^{0}(\mathbb{R}) \times Q, f(x, e, q)=$ $f_{2}(x, q), g(x, e, q)=g_{2}(x, q)$ and $e_{(e, q)}=e$. On the existence solution of $(\mathcal{G}, e, q)$ in $\mathbb{E}^{0}(\mathbb{R})$, we establish the following result. 
Corollary 5.2 Let $\rho \in \mathcal{U}_{T}$. Under the following assumptions:

(A8) $f_{2}, g_{2} \in C^{1}(\mathbb{R} \times Q, \mathbb{R})$,

(A9) $g_{2}(0,0)=0$,

(A10) $f_{2}(0,0) \neq 0$ when $f_{2}(0,0)^{2}<4 \frac{\partial g_{2}(0,0)}{\partial x}$, and $\frac{\partial g_{2}(0,0)}{\partial x} \neq 0$ when $f_{2}(0,0)^{2} \geq 4 \frac{\partial g_{2}(0,0)}{\partial x}$, there exist a neighborhood $\mathcal{W}_{2}$ of 0 in $E^{0}(\mathbb{R})$, a neighborhood $\mathcal{U}_{2}$ of 0 in $E^{2}(\mathbb{R})$, a neighborhood $\mathcal{V}_{2}$ of 0 in $Q$ and a $C^{1}$-mapping $e \mapsto \underline{x}[e, q]$ from $\mathcal{W}_{2} \times \mathcal{V}_{2}$ into $\mathcal{U}_{2}$ which satisfies the following conditions:

(i) $\underline{x}[0,0]=0$,

(ii) for all $e \in \mathcal{W}_{2}$ in $E^{0}(\mathbb{R})$ and for all $q \in \mathcal{V}_{2}, \underline{x}[e, q]$ is a solution of $(\mathcal{G}, e, q)$ in $E^{0}(\mathbb{R})$,

(iii) if $x \in \mathcal{U}_{2}$ is a solution of $(\mathcal{G}, e, q)$ with $e \in \mathcal{W}_{2}$ in $E^{0}(\mathbb{R})$ and $q \in \mathcal{V}_{2}$, then we have $x=x[e, q]$.

\section{Competing interests}

The authors declare that they have no competing interests.

\section{Author details}

'Laboratoire SAMM EA 4543, Université Paris 1 Panthéon-Sorbonne, centre P.M.F., 90 rue de Tolbiac, Paris cedex 13, 75634, France. ${ }^{2}$ Département de Mathématiques, Faculté des Sciences, Université de Skikda, Skikda, Algérie. ${ }^{3}$ Laboratoire de Mathématiques de Versailles, UMR-CNRS 8100, Université Versailles-Saint-Quentin-en-Yvelines, 45 avenue des États-Unis, Versailles cedex, 78035, France.

\section{Acknowledgements}

The authors are very pleased to contribute to this special issue in honor of Jean Mawhin, an international expert in the field of nonlinear analysis and differential equations, whose opinion is ever very important and useful to us.

\section{Received: 5 November 2012 Accepted: 8 March 2013 Published: 29 March 2013}

\section{References}

1. Blot, J, Boudjema, S: Small almost periodic and almost automorphic oscillations in forced Liénard equation. J. Abstr. Differ. Equ. Appl. 1(1), 75-85 (2010)

2. Martínez-Amores, P, Torres, PJ: Dynamics of a periodic differential equation with a singular nonlinearity of attractive type. J. Math. Anal. Appl. 202(3), 1027-1039 (1996)

3. Campos, J, Torres, PJ: On the structure of the set of bounded solutions on a periodic Liénard equation. Proc. Am. Math. Soc. 127(5), 1453-1462 (1999)

4. Cieutat, P: On the structure of the set of bounded solutions on an almost periodic Liénard equation. Nonlinear Anal. 58(7-8), 885-898 (2004)

5. Ait Dads, E, Lhachimi, L, Cieutat, P: Structure of the set of bounded solutions and existence of pseudo almost-periodic solutions of a Liénard equation. Differ. Integral Equ. 20(7), 793-813 (2007)

6. Cieutat, P, Fatajou, S, N'Guérékata, GM: Bounded and almost automorphic solutions of a Liénard equation with a singular nonlinearity. Electron. J. Qual. Theory Differ. Equ. 21, 1-15 (2008)

7. Blot, J, Cieutat, P, Mawhin, J: Almost-periodic oscillations of monotone second-order systems. Adv. Differ. Equ. 2(5), 693-714 (1997)

8. Zaidman, S: Almost-Periodic Functions in Abstract Spaces. Pitman, Marshfield (1985)

9. N'Guérékata, GM: Almost Automorphic and Almost Periodic Functions in Abstract Spaces. Kluwer Academic, New York (2001)

10. Lizama, C, N'Guérékata, GM: Bounded mild solutions for semilinear integro differential equations in Banach spaces. Integral Equ. Oper. Theory 68(2), 207-227 (2010)

11. Diagana, T: Pseudo Almost Periodic Functions in Banach Spaces. Nova Science Publishers, New York (2007)

12. Zhang, C: Almost Periodic Type Functions and Ergodicity. Science Press, Beijing (2003)

13. Liang, J, Zhang, J, Xiao, T-J: Composition of pseudo almost automorphic and asymptotically almost automorphic functions. J. Math. Anal. Appl. 340(2), 1493-1499 (2008)

14. Xiao, T-J, Liang, J, Zhang, J: Pseudo almost automorphic solutions to semilinear differential equations in Banach spaces. Semigroup Forum 76(3), 518-524 (2008)

15. Blot, J, Cieutat, P, Ezzinbi, K: New approach for weighed pseudo-almost periodic functions under the light of measure theory, basic results and applications. Appl. Anal. (2011). doi:10.1080/00036811.2011.628941

16. Blot, J, Cieutat, P, Ezzinbi, K: Measure theory and pseudo almost automorphic functions: new developments and applications. Nonlinear Anal. 75(4), 2426-2447 (2012)

17. Diagana, T: Weighted pseudo almost periodic functions and applications. C. R. Math. Acad. Sci. Paris 343(10), 643-646 (2006)

18. Diagana, T: Weighted pseudo-almost periodic solutions to some differential equations. Nonlinear Anal. 68(8), 2250-2260 (2008)

19. Blot, J, Mophou, GM, N'Guérékata, GM, Pennequin, D: Weighted pseudo almost automorphic functions and applications to abstract differential equations. Nonlinear Anal. 71(3-4), 903-909 (2009) 
20. Daleckii, JL, Krein, MG: Stability of Solutions of Differential Equations in Banach Space. Translations of Mathematical Monographs, vol. 43. Am. Math. Soc., Providence (1974)

21. Prüss, J: On the spectrum of $C_{0}$-semigroups. Trans. Am. Math. Soc. 284(2), 847-857 (1984)

22. Blot, J, Cieutat, P, N'Guérékata, GM, Pennequin, D: Superposition operators between various spaces of almost periodic function spaces and applications. Commun. Math. Anal. 6(1), 42-70 (2009)

23. Blot, J, Cartigny, P: Bounded solutions and oscillations of concave Lagrangian systems in presence of a discount rate. Z. Anal. Anwend. 14(4), 731-750 (1995)

24. Cieutat, P, Fatajou, S, N'Guérékata, GM: Composition of pseudo almost periodic and pseudo almost automorphic functions and applications to evolution equations. Appl. Anal. 89(1), 11-27 (2010)

25. Cartan, H: Cours de calcul différentiel. Hermann, Paris (1977)

doi:10.1186/1687-2770-2013-66

Cite this article as: Blot et al.: Several kinds of oscillations in forced Liénard equations. Boundary Value Problems 2013 2013:66.

\section{Submit your manuscript to a SpringerOpen ${ }^{\circ}$ journal and benefit from:}

- Convenient online submission

- Rigorous peer review

- Immediate publication on acceptance

Open access: articles freely available online

- High visibility within the field

- Retaining the copyright to your article 